\title{
A Maass space in higher genus
}

\author{
Winfried Kohnen and Hisashi Kojima
}

\begin{abstract}
We show that for arbitrary even genus $2 n$ with $n \equiv 0,1(\bmod 4)$ the subspace of Siegel cusp forms of weight $k+n$ generated by the Ikeda lifts of elliptic cusp forms of weight $2 k$ can be characterized by certain simple relations among the Fourier coefficients. These generalize the classical Maass relations in genus 2.
\end{abstract}

\section{Introduction}

For positive integers $n$ and $k$, let $S_{k}\left(\Gamma_{n}\right)$ be the space of Siegel cusp forms of weight $k$ with respect to the full Siegel modular group $\Gamma_{n}:=S p_{n}(\mathbb{Z}) \subset G L_{2 n}(\mathbb{Z})$ of genus $n$.

Suppose that $n=2$ and $k$ is even. Then as is well known there exists a certain Hecke-invariant subspace $S_{k}^{*}\left(\Gamma_{2}\right) \subset S_{k}\left(\Gamma_{2}\right)$ called the Maass space which is Hecke-equivariantly isomorphic to $S_{2 k-2}\left(\Gamma_{1}\right)$ ('Saito-Kurokawa lift'). If $F \in S_{k}^{*}\left(\Gamma_{2}\right)$ is a Hecke eigenform, then its spinor zeta function is equal to

$$
\zeta(s-k+1) \zeta(s-k+2) L(f, s),
$$

where $L(f, s)$ is the Hecke $L$-function of a normalized Hecke eigenform $f \in S_{2 k-2}\left(\Gamma_{1}\right)$. As is easy to see by a formal calculation and is well known, the standard zeta function of $F$ then equals

$$
\zeta(s) L(f, s+k-1) L(f, s+k-2) .
$$

The space $S_{k}^{*}\left(\Gamma_{2}\right)$ can be explicitly described as the image of a certain linear map from the "plus subspace' $S_{k-\frac{1}{2}}^{+}$of the space of cusp forms of weight $k-\frac{1}{2}$ and level 4 to $S_{k}\left(\Gamma_{2}\right)$ and can also be characterized in terms of certain linear relations among the Fourier coefficients of the forms ('Maass relations'). This was intensively studied in the late 1970s and early 1980s by Andrianov, Eichler and Zagier, Maass, Saito and Kurokawa, Zagier and others; for a survey of some of the results, see e.g. [EZ85, ch. II, §6].

Recently, in a significant breakthrough, Ikeda [Ike01] generalized the Saito-Kurokawa lift to higher genus. More precisely, for $f \in S_{2 k}\left(\Gamma_{1}\right)$ a normalized Hecke eigenform and $n \equiv k(\bmod 2)$, he explicitly constructed a Hecke eigenform $F \in S_{k+n}\left(\Gamma_{2 n}\right)$ such that its standard zeta function is equal to

$$
\zeta(s) \prod_{j=1}^{2 n} L(f, s+k+n-j),
$$

where again $L(f, s)$ is the Hecke $L$-function of $f$. The existence of such an $F$ was previously conjectured by Duke and Imamoglu.

In [Koh02], generalizing the corresponding result in genus 2, one of the authors gave an explicit linear version of Ikeda's lifting map as a map from $S_{k+\frac{1}{2}}^{+}$to $S_{k+n}\left(\Gamma_{2 n}\right)$ where $S_{k+\frac{1}{2}}^{+}$again denotes the 'plus subspace' of forms of weight $k+\frac{1}{2}$ and level 4 .

Received 28 October 2003, accepted in final form 26 February 2004, published online 10 February 2005. 2000 Mathematics Subject Classification 11F46.

Keywords: Siegel modular forms, Maass space.

This journal is (C) Foundation Compositio Mathematica 2005. 


\section{W. Kohnen And H. KoJima}

In [Koh02] a conjectural description of the image of this lifting map in terms of conditions on the Fourier coefficients was also given. In the case $n=1$, this condition comes down to saying that the $T$ th Fourier coefficients of a form in the Maass space, where $T$ is a rational, half-integral, symmetric, positive definite $(2,2)$-matrix, for $T$ primitive depend only on the discriminant of $T$ (see $[$ Koh02, $\S 8$, Proposition 4]). Note that in the latter case this description holds true and in fact is a well-known classical result of Zagier [Zag81].

The main purpose of this paper is to prove the conjecture of [Koh02] for all $n$ with $n \equiv 0,1$ $(\bmod 4)$ (Theorem 3$)$ and as a consequence in these cases give a characterization of the image of the lifting in terms of certain linear relations among the Fourier coefficients, generalizing the classical Maass relations (Corollary 4).

The proof proceeds by using certain specific results of [Koh02] on the Fourier coefficients of Ikedalifted forms combined with projecting a Siegel cusp form to one of its Fourier-Jacobi coefficients $\phi_{T_{0}}$ of index $T_{0}$, where $T_{0}$ is a certain special matrix of size $2 n-1$ and producing forms in fact in

$S_{k+\frac{1}{2}}^{+}$in this way, analogously to the case of genus 2. Note that such a proof was partly suggested in $[$ Koh02].

The assumption $n \equiv 0,1(\bmod 4)$ seems to be essential to our arguments, since the existence of $T_{0}$ can be guaranteed only in the latter cases. Of course, this does not mean that our proof cannot be generalized to the cases $n \equiv 2,3(\bmod 4)$ by modifying the arguments slightly in a somewhat different direction.

In $\S 2$, we will recall several facts on certain polynomials attached to rational, symmetric matrices of even size that enter in connection with Ikeda's lifting map. In $\S 3$, we will recall Ikeda's lifting theorem and its linear version given in [Koh02]. Section 4 contains the statement of our results, while their proofs are given in $\S 5$.

Notation. If $A$ and $B$ are matrices of appropriate sizes over a commutative $\operatorname{ring} R$, we put $A[B]:=$ $B^{\prime} A B$ where $B^{\prime}$ is the transpose of $B$.

If $A$ and $B$ are square matrices over $R$, we often write $A \oplus B$ for the diagonal block matrix

$$
\left(\begin{array}{ll}
A & 0 \\
0 & B
\end{array}\right) \text {. }
$$

If $A$ is a square matrix over $R$, we call $A$ even if all its diagonal elements are in $2 R$. If $R$ is an integral domain of characteristic different from 2 , the matrix $\frac{1}{2} A$ then will be called half-integral.

We denote by $R^{(n, 1)}$ the set of column vectors of length $n$ with components in $R$.

If a real, symmetric matrix $T$ is positive definite, we write $T>0$.

For a positive integer $k$, we let $S_{k+\frac{1}{2}}^{+}$be the space of cusp forms of weight $k+\frac{1}{2}$ and level 4 having a Fourier expansion of the form

$$
\sum_{m \geqslant 1,(-1)^{k} m \equiv 0,1(\bmod 4)} c(m) e^{2 \pi i m z}
$$

(where $z \in \mathcal{H}=$ the complex upper half-plane) [Koh80], [Shi73].

If $F \in S_{k}\left(\Gamma_{n}\right)$ is a Siegel cusp form of weight $k$ and genus $n$, we write its Fourier expansion in the form

$$
F(Z)=\sum_{T>0} A(T) e^{2 \pi i \operatorname{tr}(T Z)}
$$

(where $Z \in \mathcal{H}_{n}=$ the Siegel upper half-space of genus $n$ ), where $T$ runs over all rational, half-integral, symmetric, positive definite matrices of size $n$. 


\section{A MaAss SPACE IN Higher GenUs}

\section{Preliminaries}

Let $T \in M_{2 n}(\mathbb{Q})$ be a rational, half-integral, symmetric, non-degenerate matrix of size $2 n$. We denote by

$$
D_{T}:=(-1)^{n} \operatorname{det}(2 T)
$$

the discriminant of $T$. Then $D_{T} \equiv 0,1(\bmod 4)$ and we write $D_{T}=D_{T, 0} f_{T}^{2}$ with $D_{T, 0}$ the corresponding fundamental discriminant and $f_{T} \in \mathbb{N}$.

Let us fix a prime $p$. Recall that one defines the local singular series of $T$ at $p$ by

$$
b_{p}(T ; s):=\sum_{R} \nu_{p}(R)^{-s} \mathrm{e}_{p}(\operatorname{tr}(T R)) \quad(s \in \mathbb{C})
$$

where $R$ runs over all symmetric $(2 n, 2 n)$-matrices with entries in $\mathbb{Q}_{p} / \mathbb{Z}_{p}$ and $\nu_{p}(R)$ is a power of $p$ equal to the product of denominators of elementary divisors of $R$. Furthermore, for $x \in \mathbb{Q}_{p}$ we have put $\mathrm{e}_{p}(x):=e^{2 \pi i x^{\prime}}$ where $x^{\prime}$ denotes the fractional part of $x$.

As is well known, $b_{p}(T ; s)$ is a product of two polynomials in $p^{-s}$ with coefficients in $\mathbb{Z}$. More precisely, one has

$$
b_{p}(T ; s)=\gamma_{p}\left(T ; p^{-s}\right) F_{p}\left(T ; p^{-s}\right)
$$

where

$$
\gamma_{p}(T ; X):=(1-X)\left(1-\left(\frac{D_{T, 0}}{p}\right) p^{n} X\right)^{-1} \prod_{j=1}^{n}\left(1-p^{2 j} X^{2}\right)
$$

and $F_{p}(T ; X) \in \mathbb{Z}[X]$ has constant term 1 .

A fundamental result of Katsurada [Kat99] states that the Laurent polynomial

$$
\tilde{F}_{p}(T ; X):=X^{-\operatorname{ord}_{p} f_{T}} F_{p}\left(T ; p^{-n-1 / 2} X\right)
$$

is symmetric, i.e.

$$
\tilde{F}_{p}(T ; X)=\tilde{F}_{p}\left(T ; X^{-1}\right) .
$$

If $p$ does not divide $f_{T}$, then $F_{p}(T ; X)=\tilde{F}_{p}(T ; X)=1$.

Denote by $V=\left(\mathbb{F}_{p}^{2 n}, q\right)$ the quadratic space over $\mathbb{F}_{p}$ where $q$ is the quadratic form obtained from the quadratic form $x \mapsto T[x]\left(x \in \mathbb{Z}_{p}^{2 n}\right)$ by reducing modulo $p$. We denote by $\langle x, y\rangle:=$ $q(x+y)-q(x)-q(y)\left(x, y \in \mathbb{F}_{p}^{2 n}\right)$ the associated bilinear form and let

$$
R(V):=\left\{x \in \mathbb{F}_{p}^{2 n} \mid\langle x, y\rangle=0 \forall y \in \mathbb{F}_{p}^{2 n}, q(x)=0\right\}
$$

be the radical of $V$. We put $s_{p}:=s_{p}(T)=\operatorname{dim} R(V)$ and denote by $W$ an orthogonal complementary subspace of $R(V)$.

According to [Kit84], one defines a polynomial by

$$
H_{n, p}(T ; X):= \begin{cases}1, & \text { if } s_{p}=0, \\ \prod_{j=1}^{\left[\left(s_{p}-1\right) / 2\right]}\left(1-p^{2 j-1} X^{2}\right), & \text { if } s_{p}>0, s_{p} \text { odd } \\ \left(1+\lambda_{p}(T) p^{\left(s_{p}-1\right) / 2} X\right) \prod_{j=1}^{\left[\left(s_{p}-1\right) / 2\right]}\left(1-p^{2 j-1} X^{2}\right), & \text { if } s_{p}>0, s_{p} \text { even }\end{cases}
$$

where for $s_{p}$ even we have put

$$
\lambda_{p}(T):= \begin{cases}1, & \text { if } W \text { is a hyperbolic subspace or } s_{p}=2 n \\ -1, & \text { otherwise }\end{cases}
$$




\section{W. Kohnen And H. KoJima}

According to [Koh02], for $\mu \in \mathbb{Z}, \mu \geqslant 0$, define $\rho_{T}\left(p^{\mu}\right)$ by

$$
\sum_{\mu \geqslant 0} \rho_{T}\left(p^{\mu}\right) X^{\mu}:= \begin{cases}\left(1-X^{2}\right) H_{n, p}(T ; X), & \text { if } p \mid f_{T}, \\ 1, & \text { otherwise. }\end{cases}
$$

We extend the function $\rho_{T}$ multiplicatively to the whole of $\mathbb{N}$ by defining

$$
\sum_{a \geqslant 1} \rho_{T}(a) a^{-s}:=\prod_{p \mid f_{T}}\left(\left(1-p^{-2 s}\right) H_{n, p}\left(T ; p^{-s}\right)\right) .
$$

It follows from the definitions that $\sqrt{a} \rho_{T}(a)$ is an integer.

Finally, let

$$
\mathcal{D}(T):=G L_{2 n}(\mathbb{Z}) \backslash\left\{G \in M_{2 n}(\mathbb{Z}) \cap G L_{2 n}(\mathbb{Q}) \mid T\left[G^{-1}\right] \text { half-integral }\right\},
$$

where $G L_{2 n}(\mathbb{Z})$ operates by left-multiplication. Then $\mathcal{D}(T)$ is finite as is easy to see. For $a \in \mathbb{N}$ with $a \mid f_{T}$ put

$$
\phi(a ; T):=\sqrt{a} \sum_{d^{2} \mid a} \sum_{G \in \mathcal{D}(T),|\operatorname{det}(G)|=d} \rho_{T\left[G^{-1}\right]}\left(\frac{a}{d^{2}}\right) .
$$

Note that on the right-hand side of (1) we have $\left(a / d^{2}\right) \mid f_{T\left[G^{-1}\right]}$ and that $\phi(a ; T) \in \mathbb{Z}$ for all $a$.

\section{Ikeda's lifting map}

Let $f$ be a normalized Hecke eigenform in $S_{2 k}\left(\Gamma_{1}\right)$. For a prime $p$, let $\lambda(p)$ and $\alpha_{p}$ be the $p$ th Fourier coefficient and the Satake $p$-parameter of $f$, respectively. Thus

$$
1-\lambda(p) X+p^{2 k-1} X^{2}=\left(1-p^{k-1 / 2} \alpha_{p} X\right)\left(1-p^{k-1 / 2} \alpha_{p}^{-1} X\right) .
$$

Note that $\alpha_{p}$ is determined only up to inversion.

Let

$$
g=\sum_{m \geqslant 1,(-1)^{k} m \equiv 0,1(\bmod 4)} c(m) e^{2 \pi i m z} \quad(z \in \mathcal{H})
$$

be a Hecke eigenform in $S_{k+\frac{1}{2}}^{+}$which corresponds to $f$ under the Shimura correspondence [Koh80], [Shi73].

Let $n \in \mathbb{N}$ with $n \equiv k(\bmod 2)$. For $T$ a rational, half-integral, symmetric, positive definite matrix of size $2 n$, define

$$
a_{f}(T):=c\left(\left|D_{T, 0}\right|\right) f_{T}^{k-1 / 2} \prod_{p \mid f_{T}} \tilde{F}_{p}\left(T ; \alpha_{p}\right),
$$

where we have used the notation explained in $\S 2$. Note that for $n$ and $k$ of the same parity, $(-1)^{k} D_{T, 0}>0$.

THEOREM 1 [Ike01]. The function

$$
F(Z):=\sum_{T>0} a_{f}(T) e^{2 \pi i \operatorname{tr}(T Z)} \quad\left(Z \in \mathcal{H}_{2 n}\right),
$$

where $T$ runs over all rational, half-integral, symmetric, positive definite matrices of size $2 n$, is a cuspidal Siegel-Hecke eigenform of weight $k+n$ with respect to $\Gamma_{2 n}$.

Remarks. (a) Ikeda [Ike01] further proves that the standard zeta function of $F$ is given by

$$
\zeta(s) \prod_{j=1}^{2 n} L(f, s+k+n-j)
$$

where $L(f, s)$ is the Hecke $L$-function of $f$. We will not use this fact here. 


\section{A MaAss SPACE IN HIGHER GENUS}

(b) We note that it is implicit in Ikeda's theorem (respectively its proof) that his lift is injective. Indeed, if $f_{1}$ and $f_{2}$ are different normalized eigenforms in $S_{2 k}\left(\Gamma_{1}\right)$, then their $p$-eigenvalues are different for at least one prime $p$. Hence their lifts are in different eigenspaces for the local Hecke algebra at $p$ in genus $2 n$ and therefore are orthogonal with respect to the Petersson scalar product.

TheOREM 2 [Koh02]. With the notation of $\S 2$, one has

$$
a_{f}(T)=\sum_{a \mid f_{T}} a^{k-1} \phi(a ; T) c\left(\frac{\left|D_{T}\right|}{a^{2}}\right) .
$$

\section{A characterization of the image of Ikeda's lifting map}

We denote by $S_{k+n}^{*}\left(\Gamma_{2 n}\right)$ the image of the linear mapping $I_{k, n}$ from $S_{k+\frac{1}{2}}^{+}$to $S_{k+n}\left(\Gamma_{2 n}\right)$ defined by (2). (Note that this notation differs from the one used in $[\mathrm{Koh02,} \S 8$, iv].)

Our main result is the following.

Theorem 3. Suppose that $n \equiv 0,1(\bmod 4)$ and let $k \in \mathbb{N}$ with $n \equiv k(\bmod 2)$. Let $F \in S_{k+n}\left(\Gamma_{2 n}\right)$ with Fourier coefficients $A(T)$. Then the following assertions are equivalent:

(i) $F \in S_{k+n}^{*}\left(\Gamma_{2 n}\right)$;

(ii) there exist complex numbers $c(m)\left(\right.$ with $m \in \mathbb{N}$, and $\left.(-1)^{k} m \equiv 0,1(\bmod 4)\right)$ such that

$$
A(T)=\sum_{a \mid f_{T}} a^{k-1} \phi(a ; T) c\left(\frac{\left|D_{T}\right|}{a^{2}}\right)
$$

for all $T$.

Remarks. (a) The statement of Theorem 3 was conjectured in [Koh02, § 8, iv)].

(b) According to $[\mathrm{Koh} 02, \S 8$, iv)] the condition in (ii) is also equivalent to saying that $A(T)=$ $A(\tilde{T})$ whenever $D_{T}=D_{\tilde{T}}$ and $\phi(a ; T)=\phi(a ; \tilde{T})$ for all $a \mid f_{T}$.

To state our second result, recall [CS88, ch. 15, $\S 8.2$, table 15.5] that for each $g \in \mathbb{N}$ there exists exactly one genus of integral, even, symmetric matrices $S$ of size $g$ with determinant equal to 2 . A matrix in this genus is positive definite if and only if $g \equiv \pm 1(\bmod 8)$, and in this case as a representative we can take

$$
S_{0}:= \begin{cases}E_{8}^{\oplus(g-1) / 8} \oplus 2, & \text { if } g \equiv 1(\bmod 8), \\ E_{8}^{\oplus(g-7) / 8} \oplus E_{7}, & \text { if } g \equiv-1(\bmod 8),\end{cases}
$$

where (by abuse of language) $E_{8}$ and $E_{7}$ denote the Gram matrices of the $E_{8}$ - and $E_{7}$-root lattices, respectively.

Explicitly, recall that

$$
E_{8}=\left(\begin{array}{rrrrrrrr}
2 & 0 & -1 & 0 & 0 & 0 & 0 & 0 \\
0 & 2 & 0 & -1 & 0 & 0 & 0 & 0 \\
-1 & 0 & 2 & -1 & 0 & 0 & 0 & 0 \\
0 & -1 & -1 & 2 & -1 & 0 & 0 & 0 \\
0 & 0 & 0 & -1 & 2 & -1 & 0 & 0 \\
0 & 0 & 0 & 0 & -1 & 2 & -1 & 0 \\
0 & 0 & 0 & 0 & 0 & -1 & 2 & -1 \\
0 & 0 & 0 & 0 & 0 & 0 & -1 & 2
\end{array}\right)
$$

and $E_{7}$ is the upper left $(7,7)$-submatrix of $E_{8}$. 


\section{W. Kohnen And H. KoJima}

The matrix $E_{8}$ is unimodular and $E_{7}$ has determinant equal to 2 .

Suppose that $n \equiv 0,1(\bmod 4)$ and define $S_{0}$ by the right-hand side of $(3)$, with $g:=2 n-1$.

For $m \in \mathbb{N}$ with $(-1)^{n} m \equiv 0,1(\bmod 4)$ define a rational, half-integral, symmetric, positive definite matrix $T_{m}$ of size $2 n$ by

$$
T_{m}:= \begin{cases}\left(\begin{array}{cc}
\frac{1}{2} S_{0} & 0 \\
0 & m / 4
\end{array}\right), & \text { if } m \equiv 0(\bmod 4), \\
\left(\begin{array}{cc}
\frac{1}{2} S_{0} & \frac{1}{2} e_{2 n-1} \\
\frac{1}{2} e_{2 n-1}^{\prime} & {\left[m+2+(-1)^{n}\right] / 4}
\end{array}\right), & \text { if } m \equiv(-1)^{n}(\bmod 4),\end{cases}
$$

where $e_{2 n-1}=(0, \ldots, 0,1)^{\prime} \in \mathbb{Z}^{(2 n-1,1)}$ is the usual standard column vector.

Observe that the matrices on the right-hand side of (4) are indeed positive definite. This is obvious except for the case $n \equiv 0(\bmod 4), m \equiv 1(\bmod 4)$. In the latter case, the Jacobi decomposition shows that

$$
\begin{aligned}
\operatorname{det}\left(2 T_{m}\right) & =2\left(\frac{m+3}{2}-E_{7}^{-1}\left[e_{7}\right]\right) \\
& =m
\end{aligned}
$$

since $E_{7}^{-1}\left[e_{7}\right]=\frac{3}{2}$. Our claim follows from this.

We note that

$$
\operatorname{det}\left(2 T_{m}\right)=m
$$

for all $m$.

Corollary 4 ('Maass relations'). Under the same assumptions as in Theorem 3 and with the above notation, the following assertions are equivalent:

(i) $F \in S_{k+n}^{*}\left(\Gamma_{2 n}\right)$;

(ii) for all $T$, one has

$$
A(T)=\sum_{a \mid f_{T}} a^{k-1} \phi(a ; T) A\left(T_{\left|D_{T}\right| / a^{2}}\right)
$$

Remarks. (a) That (i) implies (ii), in a slightly different formulation and with a different proof, has already been shown before by one of the authors [Koh04].

(b) If $n=1$, using the fact that

$$
\phi(a ; T)= \begin{cases}a, & \text { if } a \mid \operatorname{gcd}(\alpha, \beta, \gamma), \\ 0, & \text { otherwise }\end{cases}
$$

where

$$
T=\left(\begin{array}{cc}
\alpha & \beta / 2 \\
\beta / 2 & \gamma
\end{array}\right)
$$

(see [Koh02, Equation (32)]), one recovers the classical 'Maass relations' in genus 2.

(c) One can easily give a formulation of the statement of Corollary 4 which is valid for any matrix $S$ in the genus of $S_{0}$, by properly modifying the definition of the matrices $T_{m}$. This, of course, is consistent with the fact that the Fourier coefficients $A(T)$ of an Ikeda-lifted form by definition depend only on the genus of $T$. The more general formulation is slightly more involved and is left to the reader. We have preferred to choose $S=S_{0}$ here, since in this case the statement for higher genus formally seems to come closest to the corresponding classical statement in genus 2 . 


\section{A MaAss SPACE in Higher Genus}

\section{Proof of Theorem 3 and Corollary 4}

To prove Theorem 3, in view of Theorem 2 it is sufficient to show that (ii) implies (i).

We will use the notation explained in $\S 4$, after the statement of Theorem 3.

For convenience, let us put

$$
T_{0}:=\frac{1}{2} S_{0}
$$

Let $F(Z)\left(Z \in \mathcal{H}_{2 n}\right)$ be in $S_{k+n}\left(\Gamma_{2 n}\right)$ and denote by $\phi_{T_{0}}(\tau, z)$ (with $\tau \in \mathcal{H}$, and $z \in \mathbb{C}^{(2 n-1,1)}$ ) the Fourier-Jacobi coefficient of $F$ of index $T_{0}$, i.e. the coefficient with index $T_{0}$ in a partial Fourier expansion of $F(Z)$ with respect to $w \in \mathcal{H}_{2 n-1}$ where

$$
Z=\left(\begin{array}{ll}
w & z \\
z^{\prime} & \tau
\end{array}\right)
$$

Then $\phi_{T_{0}}$ is a Jacobi cusp form of weight $k+n$ and index $T_{0}$ with respect to the Jacobi group $\Gamma_{1} \triangleright\left(\mathbb{Z}^{(2 n-1,1)} \times \mathbb{Z}^{(2 n-1,1)}\right)$ and $\phi_{T_{0}}$ has a Fourier expansion

$$
\phi_{T_{0}}(\tau, z)=\sum_{T=\left(\begin{array}{cc}
T_{0} & r / 2 \\
r^{\prime} / 2 & N
\end{array}\right)>0} A(T) e^{2 \pi i\left(N \tau+r^{\prime} z\right)}
$$

(see [EZ85], [Ike01, §§ 7 and 8], [Shi78], [Zie89]).

As is well known, $\phi_{T_{0}}$ has an expansion in terms of Jacobi theta functions

$$
\phi_{T_{0}}(\tau, z)=\sum_{\lambda \in \Lambda} h_{\lambda}(\tau) \theta_{\lambda}(\tau, z),
$$

where $\Lambda:=S_{0}^{-1} \mathbb{Z}^{(2 n-1,1)} / \mathbb{Z}^{(2 n-1,1)}$ and where for $\lambda \in \Lambda$ one sets

$$
h_{\lambda}(\tau):=\sum_{N \in \mathbb{Z}, N-T_{0}[\lambda]>0} A\left(\left(\begin{array}{cc}
T_{0} & T_{0} \lambda \\
\lambda^{\prime} T_{0} & N
\end{array}\right)\right) e^{2 \pi i\left(N-T_{0}[\lambda]\right) \tau} \quad(\tau \in \mathcal{H})
$$

and

$$
\theta_{\lambda}(\tau, z):=\sum_{r \in \mathbb{Z}^{(2 n-1,1)}} e^{2 \pi i\left(T_{0}[r+\lambda] \tau+2(r+\lambda)^{\prime} T_{0} z\right)} \quad\left(\tau \in \mathcal{H}, z \in \mathbb{C}^{(2 n-1,1)}\right)
$$

(see [EZ85], [Ike01, §§ 7 and 8], [Shi78], [Zie89]).

We note that $|\Lambda|=2$ and that representatives can be chosen as $\lambda_{0}, \lambda_{1}$ where $\lambda_{0}$ is the zero vector and $\lambda_{1}:=S_{0}^{-1} e_{2 n-1}$. Indeed, this is trivial if $n \equiv 1(\bmod 4)$ and for $n \equiv 0(\bmod 4)$ follows from the well-known fact that $E_{7}$ is obtained from $E_{8}$ as the orthogonal complement of a vector of length 2 and 2-adically $E_{8}$ is the sum of four hyperbolic planes.

In the case $n \equiv 0(\bmod 4)$, in more elementary terms one can also argue as follows. One has

$$
\begin{aligned}
E_{7}^{-1} \mathbb{Z}^{(7,1)} / \mathbb{Z}^{(7,1)} & \simeq \prod_{p} E_{7}^{-1} \mathbb{Z}_{p}^{(7,1)} / \mathbb{Z}_{p}^{(7,1)} \\
& \simeq E_{7}^{-1} \mathbb{Z}_{2}^{(7,1)} / \mathbb{Z}_{2}^{(7,1)}
\end{aligned}
$$

since $\operatorname{det}\left(E_{7}\right)=2$. Furthermore

$$
E_{7}=\left(\begin{array}{cc}
E_{7}^{(1)} & * \\
* & 2,
\end{array}\right),
$$

where $E_{7}^{(1)}$ is of size 6 and has determinant equal to $3 \in \mathbb{Z}_{2}^{*}$; hence we see from the Jacobi decomposition that $E_{7}$ over $\mathbb{Z}_{2}$ is equivalent to $E_{7}^{(1)} \oplus \frac{2}{3}$ which implies that

$$
E_{7}^{-1} \mathbb{Z}_{2}^{(7,1)} / \mathbb{Z}_{2}^{(7,1)} \simeq \mathbb{Z} / 2 \mathbb{Z}
$$




\section{W. Kohnen And H. KoJima}

On the other hand, a direct calculation shows that the congruence $E_{7} r \equiv e_{7}(\bmod 2)$ with $r \in \mathbb{Z}^{(7,1)}$ is not solvable hence $E_{7}^{-1} e_{7}$ is not in $\mathbb{Z}^{(7,1)}$.

With the above representatives, we now find that

$$
h_{0}(\tau):=h_{\lambda_{0}}(\tau)=\sum_{N>0} A\left(\left(\begin{array}{cc}
T_{0} & 0 \\
0 & N
\end{array}\right)\right) e^{2 \pi i N \tau} \quad(\tau \in \mathcal{H})
$$

and

$$
h_{1}(\tau):=h_{\lambda_{1}}(\tau)=\sum_{N>\left[2+(-1)^{n}\right] / 4} A\left(\left(\begin{array}{cc}
T_{0} & \frac{1}{2} e_{2 n-1} \\
\frac{1}{2} e_{2 n-1}^{\prime} & N
\end{array}\right)\right) e^{2 \pi i\left(N-\left[2+(-1)^{n}\right] / 4\right) \tau} \quad(\tau \in \mathcal{H}) .
$$

We claim that the function

$$
h(\tau):=h_{0}(4 \tau)+h_{1}(4 \tau) \quad(\tau \in \mathcal{H})
$$

is in $S_{k+\frac{1}{2}}^{+}$. Indeed, from [Ike01, 9 ], [Shi78], [Zie89, ch. 3, Equations (1) and (2)] (in the case $n=1$, cf. also [EZ85, ch. II, $\S 5]$ ) one finds that

$$
\left(\begin{array}{l}
h_{0}(\tau+1) \\
h_{1}(\tau+1)
\end{array}\right)=\left(\begin{array}{cc}
1 & 0 \\
0 & -\epsilon_{n} i
\end{array}\right)\left(\begin{array}{l}
h_{0}(\tau) \\
h_{1}(\tau)
\end{array}\right)
$$

and (noting $\left.k+n-(2 n-1) / 2=k+\frac{1}{2}\right)$ that

$$
\left(\begin{array}{l}
h_{0}(-1 / \tau) \\
h_{1}(-1 / \tau)
\end{array}\right)=\frac{1+\epsilon_{n} i}{2} \tau^{k+\frac{1}{2}}\left(\begin{array}{cc}
1 & 1 \\
1 & -1
\end{array}\right)\left(\begin{array}{l}
h_{0}(\tau) \\
h_{1}(\tau)
\end{array}\right)
$$

where

$$
\epsilon_{n}:= \begin{cases}1, & \text { if } n \equiv 1(\bmod 4), \\ -1, & \text { if } n \equiv 0(\bmod 4),\end{cases}
$$

and complex powers as usual are defined by the principal value of the complex logarithm.

A formal computation similar to the case $n=1$ now easily shows that

$$
h(\tau+1)=h(\tau), \quad h\left(\frac{\tau}{4 \tau+1}\right)=(4 \tau+1)^{k+\frac{1}{2}} h(\tau) .
$$

Since

$$
\left(\begin{array}{ll}
1 & 1 \\
0 & 1
\end{array}\right) \text { and }\left(\begin{array}{ll}
1 & 0 \\
4 & 1
\end{array}\right)
$$

generate $\Gamma_{0}(4)$, we conclude that $h(\tau)$ behaves like a modular form of weight $k+\frac{1}{2}$ and level 4 . From the above transformation formulas one sees that $h(\tau)$ is cuspidal and Equations $\left(6_{1}\right)$ and $\left(6_{2}\right)$ then imply that $h(\tau)$ in fact is contained in $S_{k+\frac{1}{2}}^{+}$(note that $n \equiv k(\bmod 2)$ by assumption).

The Fourier coefficients $\tilde{c}(m)$ (where $m \in \mathbb{N}$, and $\left.(-1)^{k} m \equiv 0,1(\bmod 4)\right)$ of $h$ are given by the Fourier coefficients of the right-hand sides of Equations $\left(6_{1}\right)$ and $\left(6_{2}\right)$, with $\tau$ replaced by $4 \tau$, and with obvious identifications of $m$ and $N$. More precisely, note that for a typical matrix $T$ with $A(T)$ appearing on the right-hand sides of Equations $\left(6_{1}\right)$ and $\left(6_{2}\right)$ we have

$$
\operatorname{det}(2 T)=m= \begin{cases}4 N, & \text { if } \lambda=\lambda_{0}, \\ 4 N-\left(2+(-1)^{n}\right), & \text { if } \lambda=\lambda_{1}\end{cases}
$$

(compare (5)). We claim that each $T$ appearing in this way has the property that $s_{p}(T)=1$ for all primes $p$ such that $p \mid f_{T}$.

Since $E_{8}$ is unimodular and $\operatorname{det}\left(E_{7}\right)=2$, this readily follows if $p>2$. If $p=2$ and $2 \mid f_{T}$, then by (7) only the case $\lambda=\lambda_{0}$ matters. As was already noted before, $E_{7}$ over $\mathbb{Z}_{2}$ is equivalent to a matrix 


\section{A MaAss SPACE IN HIGHER GENUS}

$E_{7}^{(1)} \oplus \frac{2}{3}$ with $E_{7}^{(1)} \in G L_{6}\left(\mathbb{Z}_{2}\right)$. Since $s_{2}(1 \oplus N)=1$ as follows from the definitions, we therefore see that $s_{2}(T)=1$ in the case under consideration. This implies our claim.

We can now finish the proof of Theorem 3. By assumption, there exist complex numbers $c(m)$ (with $m \in \mathbb{N}$, and $\left.(-1)^{k} m \equiv 0,1(\bmod 4)\right)$ such that

$$
A(T)=\sum_{a \mid f_{T}} a^{k-1} \phi(a ; T) c\left(\frac{\left|D_{T}\right|}{a^{2}}\right)
$$

for all $T$. If we choose for $T$ one of the special matrices occurring on the right-hand sides of Equations of $\left(6_{1}\right)$ and $\left(6_{2}\right)$, then for those $T$ we have $s_{p}(T)=1$ for all primes $p$ with $p \mid f_{T}$ as was just proved. By a formal calculation, using only the definition of $\phi(a ; T)$ in exactly the same way as in the proof of [Koh02, Proposition 2, p. 801], we therefore deduce that $A(T)=c\left(\left|D_{T}\right|\right)$ for those $T$; in other words we find that

$$
\tilde{c}(m)=c(m)
$$

for all $m \in \mathbb{N}$ with $(-1)^{k} m \equiv 0,1(\bmod 4)$.

This proves Theorem 3 .

Let us now prove Corollary 4. Checking the definitions, we see that the implication (i) $\Rightarrow$ (ii) has already been proved above.

Conversely, suppose that (ii) holds. For $m \in \mathbb{N}$ with $(-1)^{k} m \equiv 0,1(\bmod 4)$, define

$$
c(m):=A\left(T_{m}\right) .
$$

Then

$$
A(T)=\sum_{a \mid f_{T}} a^{k-1} \phi(a ; T) c\left(\frac{\left|D_{T}\right|}{a^{2}}\right)
$$

for all $T$; hence by Theorem 3 we infer that $F \in S_{k+n}^{*}\left(\Gamma_{2 n}\right)$.

Remark. Note that, as a side result, we have proved that in the above notation the mapping $S_{k+n}\left(\Gamma_{2 n}\right) \rightarrow S_{k+\frac{1}{2}}^{+}, F \mapsto h$ is surjective and that its restriction to $S_{k+n}^{*}\left(\Gamma_{2 n}\right)$ is bijective with inverse given by $I_{k, n}$. Thus also in higher genus one recovers an analogue of the well-known situation in genus 2 .

\section{REFERENCES}

CS88 J. H. Conway and N. J. A. Sloane, Sphere packings, lattices and groups, Grundlehren Math. Wiss., vol. 290 (Springer, Berlin, 1988).

EZ85 M. Eichler and D. Zagier, The theory of Jacobi forms, Progress in Mathematics, vol. 55 (Birkhäuser, Boston, MA, 1985).

Ike01 T. Ikeda, On the lifting of elliptic modular forms to Siegel cusp forms of degree $2 n$, Ann. of Math. (2) 154 (2001), 641-681.

Kat99 H. Katsurada, An explicit form for Siegel series, Amer. J. Math. 121 (1999), 415-452.

Kit84 Y. Kitaoka, Dirichlet series in the theory of Siegel modular forms, Nagoya Math. J. 95 (1984), 73-84.

Koh80 W. Kohnen, Modular forms of half-integral weight on $\Gamma_{0}(4)$, Math. Ann. 248 (1980), 249-266.

Koh02 W. Kohnen, Lifting modular forms of half-integral weight to Siegel modular forms of even genus, Math. Ann. 322 (2002), 787-809.

Koh04 W. Kohnen, Linear relations between Fourier coefficients of special Siegel modular forms, Nagoya Math. J. 173 (2004), 153-161.

Shi73 G. Shimura, On modular forms of half-integral weight, Ann. of Math. (2) 97 (1973), 440-481. 


\section{A MAASS SPACE IN HIGHER GENUS}

Shi78 G. Shimura, On certain reciprocity laws for theta functions and modular forms, Acta Math. 141 (1978), 35-71.

Zag81 D. Zagier, Sur la conjecture de Saito-Kurokawa (d'après H. Maass), in Seminar of number theory (Paris, 1979-1980), Progress in Mathematics, vol. 12 (Birkhäuser, Boston, 1981), 371-394.

Zie89 C. Ziegler, Jacobi forms of higher degree, Abh. Math. Sem. Univ. Hamburg 59 (1989), 191-224.

Winfried Kohnen winfried@mathi.uni-heidelberg.de

Mathematisches Institut, Universität Heidelberg, Im Neuenheimer Feld 288, D-69120 Heidelberg, Germany

Hisashi Kojima hkojima@rimath.saitama-u.ac.jp

Department of Mathematics, Faculty of Science, Iwate University, Morioka 020, Japan

Current address: Department of Mathematics, Faculty of Science, Saitama University, Urawa 338-8570, Japan 